\title{
Damage Location Index of Shear Structures Based on Changes in First Two Natural Frequencies
}

\author{
Hien HoThu and Akira Mita \\ Mita Laboratory, Department of System Design Engineering, Keio University, 3-14-1 Hiyoshi, Kohoku-ku, Yokohama 223-8522, Japan \\ Correspondence should be addressed to Hien HoThu; thuhienjolie@gmail.com
}

Received 20 February 2014; Accepted 23 April 2014; Published 22 June 2014

Academic Editor: Yiyang Li

Copyright ( $) 2014 \mathrm{H}$. HoThu and A. Mita. This is an open access article distributed under the Creative Commons Attribution License, which permits unrestricted use, distribution, and reproduction in any medium, provided the original work is properly cited.

A method of detecting the location of damage in shear structures by using only the changes in first two natural frequencies of the translational modes is proposed. This damage detection method can determine the damage location in a shear building by using a Damage Location Index (DLI) based on two natural frequencies for undamaged and damaged states. In this study, damage is assumed to be represented by the reduction in stiffness. This stiffness reduction results in a change in natural frequencies. The uncertainty associated with system identification methods for obtaining natural frequencies is also carefully considered. Some simulations and experiments on shear structures were conducted to verify the performance of the proposed method.

\section{Introduction}

In the structural health monitoring (SHM) field, many damage detection algorithms based on the modal properties of a structure, such as modal frequencies, mode shapes, curvature mode shapes, and modal flexibilities, have been studied for several decades. However, with most algorithms, identifying the precise location and magnitude of the damage is difficult. If not completely impossible, the accuracy and reliability are not sufficient [1]. Zhao and DeWolf [2] presented a sensitivity study comparing the use of natural frequencies, mode shapes, and modal flexibilities for monitoring. Based on the fact that natural frequencies are sensitive indicators of structural integrity, the relationship between frequency changes and structural damage was discussed in a review by Salawu [3].

Many of the methods using changes in natural frequencies to detect damage were summarized by Doebling in [4]. The amount of literature is large, comprising not only applications to various structures, but also theoretical work on the use of frequency shifts for damage detection.

Besides, the trade-off relation between the number of sensors and the damage detection accuracy should be considered when installing the SHM system. A large number of sensors results in a high system cost as well as the need for enormous effort for wiring and installation. Complicated and expensive SHM systems are not feasible for most buildings [5].

Some methods have performed well at frequency-based damage identification for a few degrees of freedom. For larger engineering structures, the number of natural frequencies that can be identified is smaller than the number of structural elements. This is one of the reasons why frequency change methods have limited damage detection abilities [6]. To overcome these problems, some researchers have been using the substructure method [7], error matrix method [8], sensitivity-based method [9], combination of sensitivity and statistical-based methods [10], and so on.

Although many previous studies concluded that the frequency generally could not provide spatial information about structural changes, multiple frequency shifts may provide spatial information about structural damage if many natural frequencies are available [5]. However, in most buildings, the available number of natural frequencies is small.

The purpose of this study is to investigate a detection method that can identify the location of damage in shear structures from the changes in only two first natural frequencies. 


\section{Damage Index Based on Two First Natural Frequencies}

A multistory shear structure ( $N$-story) is modeled as a onedimensional lumped mass shear model, as shown in Figure 1. Most of the damage to the structure, such as cracks, fatigue, corrosion, and the loosening of bolted joints, manifests itself as stiffness reduction. Although the yielded structure is undamaged and may also cause reduced stiffness sometime later, in this study, we assumed that the structural damage can be directly expressed by the stiffness reduction and that the structural mass remains unchanged.

The characteristic equation for that system is written as

$$
\left[\mathbf{K}-\left(\omega^{(r)}\right)^{2} \mathbf{M}\right] \boldsymbol{\emptyset}_{r}=0
$$

where $\omega^{(r)}$ and $\emptyset_{r}$ are the $r$ th frequency and mode shape, respectively, $\mathbf{K}$ is the stiffness matrix $(N \times N)$,
$\mathbf{K}$

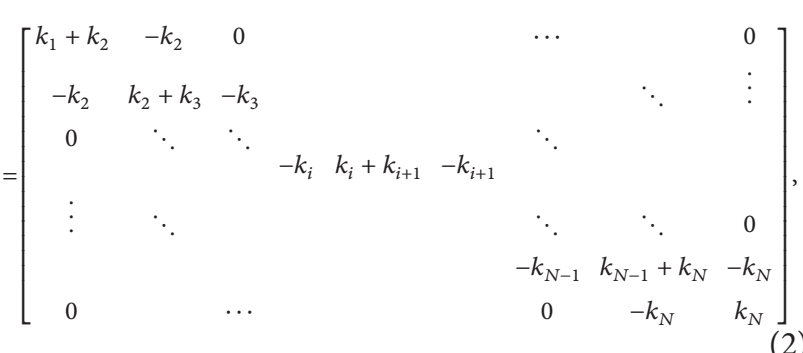

and $\mathbf{M}$ is the mass matrix $(N \times N)$,

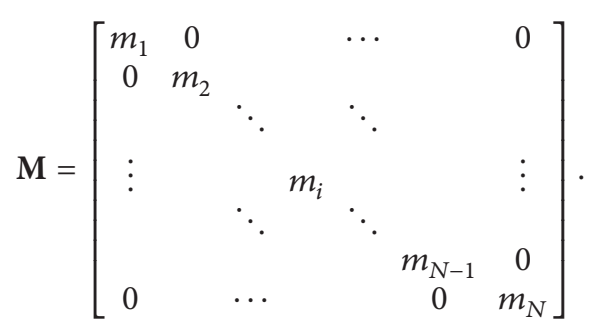

$$
\begin{aligned}
& {\left[\begin{array}{ccccccc}
\left(k_{1}+k_{2}\right)-\left(\omega^{(r)}\right)^{2} m_{1} & -k_{2} & 0 & & \cdots & \\
-k_{2} & \left(k_{2}+k_{3}\right)-\left(\omega^{(r)}\right)^{2} m_{2} & -k_{3} & & & & \\
0 & \ddots & \ddots & & & & \\
\\
0 & & & -k_{i}\left(k_{i}+k_{i+1}\right)-\left(\omega^{(r)}\right)^{2} m_{i}-k_{i+1} & & \\
\\
\vdots & & & & \ddots & \ddots & 0 \\
0 & & & & -k_{N-1}\left(k_{N-1}+k_{N}\right)-\left(\omega^{(r)}\right)^{2} m_{N-1} & -k_{N} \\
& & & & 0 & -k_{N} & k_{N}-\left(\omega^{(r)}\right)^{2} m_{N}
\end{array}\right]} \\
& \times\left[\begin{array}{c}
\emptyset_{1 r} \\
\emptyset_{2 r} \\
\vdots \\
\emptyset_{i r} \\
\vdots \\
\emptyset_{(N-1) r} \\
\emptyset_{N r}
\end{array}\right]=\left[\begin{array}{c}
0 \\
0 \\
\vdots \\
0 \\
\vdots \\
0 \\
0
\end{array}\right] .
\end{aligned}
$$

The general expression for the ith story stiffness can be obtained by solving (4):

$$
k_{i}=\frac{\left(\omega^{(r)}\right)^{2}}{\left(\emptyset_{i r}-\emptyset_{(i-1) r}\right)} \sum_{j=i}^{N} m_{j} \emptyset_{j r} .
$$

The squared $r$ th frequency in the undamaged state is

$$
\left(\omega^{(r)}\right)^{2}=k_{i} \frac{\left(\emptyset_{i r}-\emptyset_{(i-1) r}\right)}{\sum_{j=i}^{N} m_{j} \emptyset_{j r}} .
$$

Moreover, the change in the $r$ th mode natural frequency when damage occurs in the $i$ th story is defined as

$$
\Delta_{i}^{(r)}=\frac{\omega^{(r)}-\omega_{i}^{(r)}}{\omega^{(r)}},
$$

where $\omega^{(r)}$ and $\omega_{i}^{(r)}$ are the $r$ th frequency of the undamaged and $i$ th story damaged states, respectively.

As damage normally causes a decrease in natural frequencies, $\Delta_{i}^{(r)}$ are positive when damage occurs. This frequency change ratio $\Delta_{i}^{(r)}$ may vary depending on the damage location and quantification.

In [5], Mita and Hagiwara gave a different way to obtain $\Delta_{i}^{(r)}$ in (7) above as

$$
\Delta_{i}^{(r)}=\frac{\Delta k_{i}}{2\left(\omega^{(r)}\right)^{2}}\left(\emptyset_{i r}-\emptyset_{(i-1) r}\right)^{2},
$$

where $\Delta k_{i}$ is the stiffness reduction at the $i$ th story and $\emptyset_{i r}$ and $\emptyset_{(i-1) r}$ are the $r$ th mode shapes at the $i$ th and $(i-1)$ th stories, respectively. 
This frequency change $\Delta_{i}^{(r)}$ calculated by (8) may vary depending on the damage location and quantification. It should be noted that, for each natural frequency, there are some locations where the frequency is most sensitive to the damage, while there are other locations where the damage has little influence on the frequency.

Substituting (6) into (8) gives

$$
\Delta_{i}^{(r)}=\frac{\Delta k_{i}}{2 k_{i}}\left(\emptyset_{i r}-\emptyset_{(i-1) r}\right) \sum_{j=i}^{N} m_{j} \emptyset_{j r} .
$$

Similarly, frequency change $\Delta_{i}^{(s)}$ is also obtained as

$$
\Delta_{i}^{(s)}=\frac{\Delta k_{i}}{2 k_{i}}\left(\emptyset_{i s}-\emptyset_{(i-1) s}\right) \sum_{j=i}^{N} m_{j} \emptyset_{j s} .
$$

The sum of those changes is calculated as

$$
\begin{aligned}
\Delta_{i}^{(r)} & +\Delta_{i}^{(s)} \\
& =\frac{\Delta k_{i}}{2 k_{i}}\left[\left(\emptyset_{i r}-\emptyset_{(i-1) r}\right) \sum_{j=i}^{N} m_{j} \emptyset_{j r}+\left(\emptyset_{i s}-\emptyset_{(i-1) s}\right) \sum_{j=i}^{N} m_{j} \emptyset_{j s}\right] .
\end{aligned}
$$

The Damage Location Index (DLI) based on the changes in two natural frequencies of the $r$ th and $s$ th modes is written as

$$
\begin{aligned}
\mathrm{DLI} & =\frac{\Delta_{i}^{(r)}}{\Delta_{i}^{(r)}+\Delta_{i}^{(s)}} \\
& =\frac{\left(\emptyset_{i r}-\emptyset_{(i-1) r}\right) \sum_{j=i}^{N} m_{j} \emptyset_{j r}}{\left(\emptyset_{i r}-\emptyset_{(i-1) r}\right) \sum_{j=i}^{N} m_{j} \emptyset_{j r}+\left(\emptyset_{i s}-\emptyset_{(i-1) s}\right) \sum_{j=i}^{N} m_{j} \emptyset_{j s}} .
\end{aligned}
$$

From (5), we can get

$$
\begin{gathered}
\frac{\left(\omega^{(r)}\right)^{2}}{\left(\emptyset_{i r}-\emptyset_{(i-1) r}\right)} \sum_{j=i}^{N} m_{j} \emptyset_{j r}=\frac{\left(\omega^{(s)}\right)^{2}}{\left(\emptyset_{i s}-\emptyset_{(i-1) s}\right)} \sum_{j=i}^{N} m_{j} \emptyset_{j s} \\
\Longrightarrow\left(\emptyset_{i s}-\emptyset_{(i-1) s}\right)=\frac{\left(\omega^{(s)}\right)^{2}}{\left(\omega^{(r)}\right)^{2}} \frac{\sum_{j=i}^{N} m_{j} \emptyset_{j s}}{\sum_{j=i}^{N} m_{j} \emptyset_{j r}}\left(\emptyset_{i r}-\emptyset_{(i-1) r}\right) .
\end{gathered}
$$

Substituting (14) into (12),

$$
\begin{aligned}
\mathrm{DLI} & =\frac{\Delta_{i}^{(r)}}{\Delta_{i}^{(r)}+\Delta_{i}^{(s)}} \\
& =\frac{1}{1+\left(\left(\omega^{(s)}\right)^{2} /\left(\omega^{(r)}\right)^{2}\right)\left(\left(\sum_{j=i}^{N} m_{j} \emptyset_{j s}\right) / \sum_{j=i}^{N} m_{j} \emptyset_{j r}\right)^{2}} .
\end{aligned}
$$

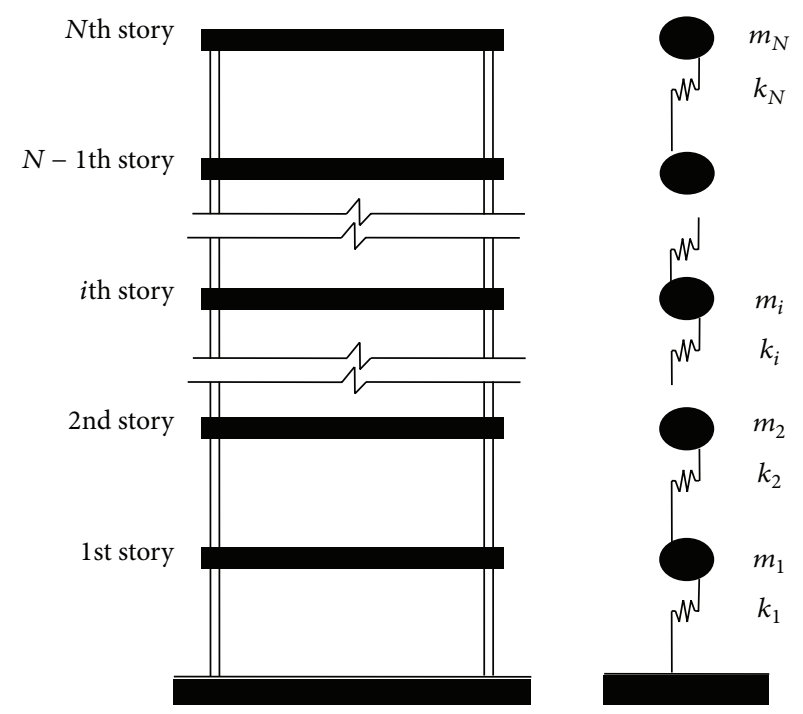

Figure 1: Simplified structural model with $N$ degrees of freedom.

From (15), this DLI value depends on the location of damage $i$ and the number of degrees of freedom $N$ :

$$
\begin{aligned}
& \frac{\sum_{j=i}^{N} m_{j} \emptyset_{j s}}{\sum_{j=i}^{N} m_{j} \emptyset_{j r}} \\
& \quad=\frac{m_{i} \emptyset_{i s}+m_{i+1} \emptyset_{(i+1) s}+\cdots+m_{N} \emptyset_{N s}}{m_{i} \emptyset_{i r}+m_{i+1} \emptyset_{(i+1) r}+\cdots+m_{N} \emptyset_{N r}} \quad \text { for } i=1,2, \ldots, N .
\end{aligned}
$$

It is assumed that the natural frequency of only the two first modes is available when damage occurs in the $i$ th story.

Thus, the DLI is proposed as a stable index defined as

$$
\begin{aligned}
\mathrm{DLI} & =\frac{\Delta_{i}^{(1)}}{\Delta_{i}^{(1)}+\Delta_{i}^{(2)}} \\
& =\frac{1}{1+\left(\left(\omega^{(2)}\right)^{2} /\left(\omega^{(1)}\right)^{2}\right)\left(\left(\sum_{j=i}^{N} m_{j} \emptyset_{j 2}\right) / \sum_{j=i}^{N} m_{j} \emptyset_{j 1}\right)^{2}} .
\end{aligned}
$$

As the change in mode shapes is relatively small, DLI will be stable regardless of the damage level.

Moreover, the modal parameters are often sensitive to various environmental conditions such as temperature, humidity, and excitation amplitude. The effect of environmental conditions or excitation amplitude is treated as "noise" in the simulation, so we should obtain the confidence interval for modal parameters. According to some previous research on the estimation of natural frequencies [11-17], the standard deviation of a few first modes of natural frequencies is less than $1 \%$. Hence, assuming that the standard deviation of the natural frequency is $\sigma_{\omega}=1 \%$, we will discuss the reliability of our proposed method on the basis of the theory in [18]. 
The standard deviation of the difference in natural frequencies is given by

$$
\sigma_{\left(\omega^{(1)}-\omega_{i}^{(1)}\right)}=\sqrt{\left(\sigma_{\omega^{(1)}}\right)^{2}+\left(\sigma_{\omega_{i}^{(1)}}\right)^{2}} .
$$

And the standard deviation of the changes in the 1st frequency is given by

$$
\sigma_{\Delta^{(1)}}=\Delta^{(1)} \sqrt{\left(\frac{\sigma_{\left(\omega^{(1)}-\omega_{i}^{(1)}\right)}}{\omega^{(1)}-\omega_{i}^{(1)}}\right)^{2}+\left(\frac{\sigma_{\omega^{(1)}}}{\omega^{(1)}}\right)^{2}} .
$$

The standard deviation of the changes in the 2nd frequency $\sigma_{\Delta^{(2)}}$ is calculated similarly to $\sigma_{\Delta^{(1)}}$ in (19) as

$$
\sigma_{\Delta^{(2)}}=\Delta^{(2)} \sqrt{\left(\frac{\sigma_{\left(\omega^{(2)}-\omega_{i}^{(2)}\right)}}{\omega^{(2)}-\omega_{i}^{(2)}}\right)^{2}+\left(\frac{\sigma_{\omega^{(2)}}}{\omega^{(2)}}\right)^{2}} .
$$

The standard deviation of the sum of the changes in two first frequencies is

$$
\sigma_{\left(\Delta^{(1)}+\Delta^{(2)}\right)}=\sqrt{\left(\sigma_{\Delta^{(1)}}\right)^{2}+\left(\sigma_{\Delta^{(2)}}\right)^{2}} .
$$

The standard deviation of the proposed DLI can be calculated as

$$
\sigma_{\mathrm{DLI}}=\mathrm{DLI} \sqrt{\left(\frac{\sigma_{\Delta^{(1)}}}{\Delta^{(1)}}\right)^{2}+\left(\frac{\sigma_{\left.\Delta^{(1)}+\Delta^{(2)}\right)}}{\Delta^{(1)}+\Delta^{(2)}}\right)^{2}} .
$$

The proposed DLI values can be calculated by (17) and the intervals are chosen to be $2 \sigma_{\mathrm{DLI}}$, with $\sigma_{\mathrm{DLI}}$ obtained using (22). The DLI reliability is within $\left\langle\mathrm{DLI} \pm 2 \sigma_{\mathrm{DLI}}\right\rangle$. We will see if our method can be used to identify the damage location in a shear structure with satisfactory reliability.

For example, a five-story structure having the same mass, $m_{i}=1000$ tons, and the same stiffness, $k_{i}=1.3 \times 10^{3} \mathrm{MN} / \mathrm{m}$, for each story, will be used to explain this damage detection procedure.

The damping ratio for each mode was chosen to be $3 \%$. The data sampling frequency was $200 \mathrm{~Hz}$. The undamaged natural frequencies of the structure are $2.03 \mathrm{~Hz}, 5.91 \mathrm{~Hz}$, $9.32 \mathrm{~Hz}, 11.98 \mathrm{~Hz}$, and $13.66 \mathrm{~Hz}$ for the 1st, 2nd, 3rd, 4th, and 5th modes, respectively. The damage was introduced by reducing the story stiffness of each story by $5 \%, 10 \%, 15 \%$, $20 \%$, and $25 \%$.

The sensitivity of the changes in two first natural frequencies to the damage is shown in Figure 2. For each natural frequency, there are some locations where this frequency is the most sensitive to the damage and others where the damage has little influence on this frequency. From Figure 2, the influence of damage location on the first natural frequency decreases at higher stories; this means that the first natural frequency is most sensitive to damage at the 1st story. By contrast, the second natural frequency is the least sensitive to damage at the 2nd story.

Here, the damage was introduced by reducing the story stiffness of each story from $5 \%$ to $25 \%$. There were 21 levels

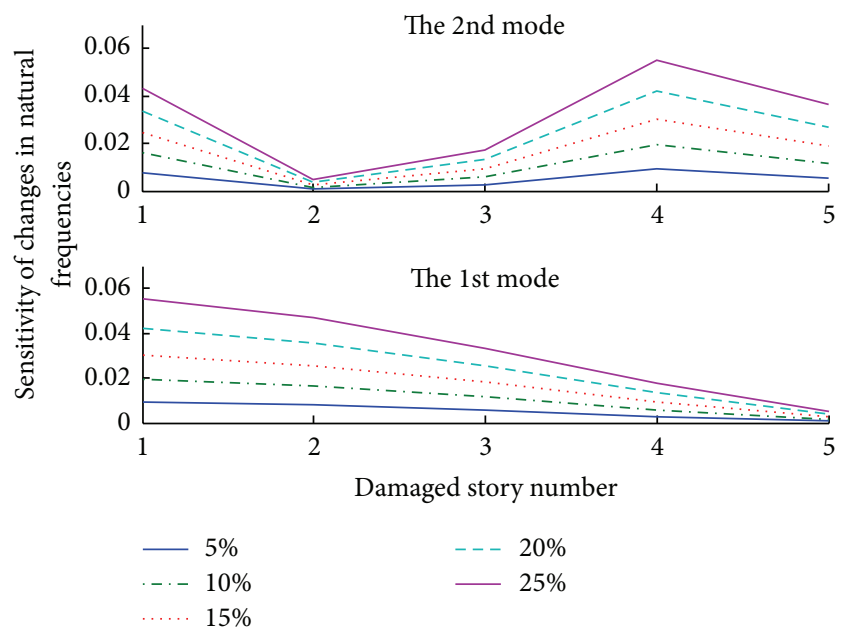

FIGURE 2: Sensitivity of changes in two first natural frequencies $\left(\Delta^{(1)}\right.$ and $\left.\Delta^{(2)}\right)$ of 5-story shear structure.

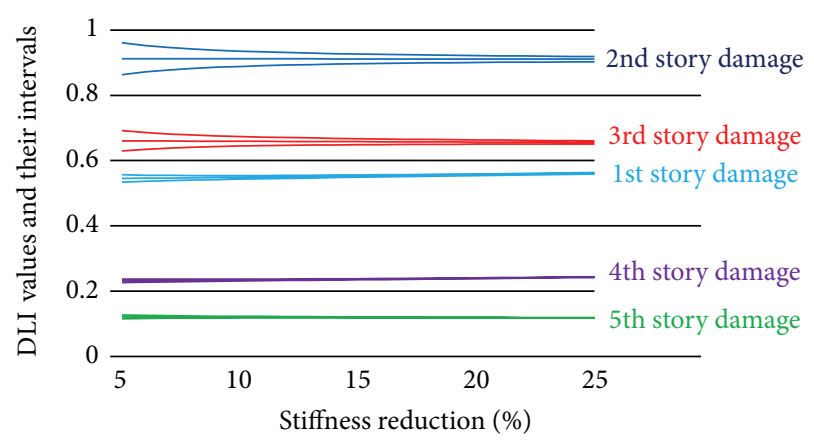

Figure 3: DLI values and their intervals within the reliability band $\pm 2 \sigma_{\text {DLI }}$ of 5 -story shear structure.

of damage in each story damage case. So $21 \times 5=105$ damage cases were examined. The DLI values of these cases were obtained by (17) and are indicated by the bold line in the middle of each color.

The standard deviations of DLI for 105 damage cases were calculated by (22). The reliability band considering $\pm 2 \sigma_{\text {DLI }}$ was also calculated and is shown by the two thin lines above and below each color. The DLI values and their confidence intervals for 5 to $25 \%$ stiffness reduction in each story indicated by the different colors are presented in Figure 3.

\section{Simulation Verification}

To show the feasibility of the proposed method with $N$ degrees of freedom, we used a three-story and an eight-story standard shear structure (same mass $m_{i}=1000$ tons and same stiffness $k_{i}=1.3 \times 10^{3} \mathrm{MN} / \mathrm{m}$ for every story). The damping ratio for each mode was chosen to be $3 \%$. The data sampling frequency was $200 \mathrm{~Hz}$. For each structure, damage was introduced by reducing the story stiffness of every story from $5 \%$ to $25 \%$. There were 21 levels of damage for each story damage case. The DLI values of these damage cases were 


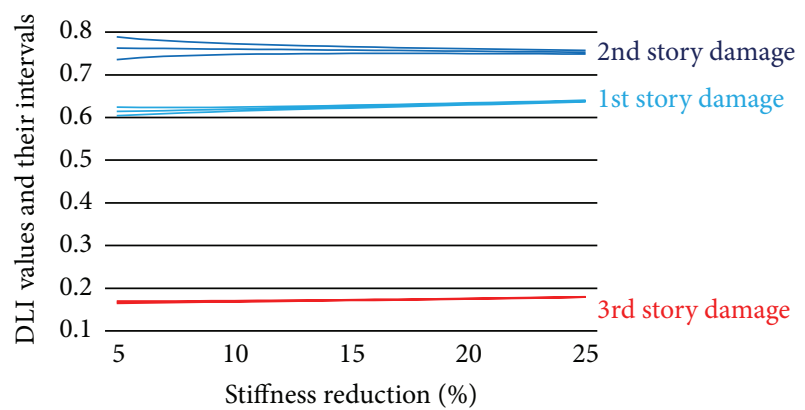

FIGURE 4: DLI values and their intervals within the reliability band $\pm 2 \sigma_{\text {DLI }}$ of 3 -story structure.

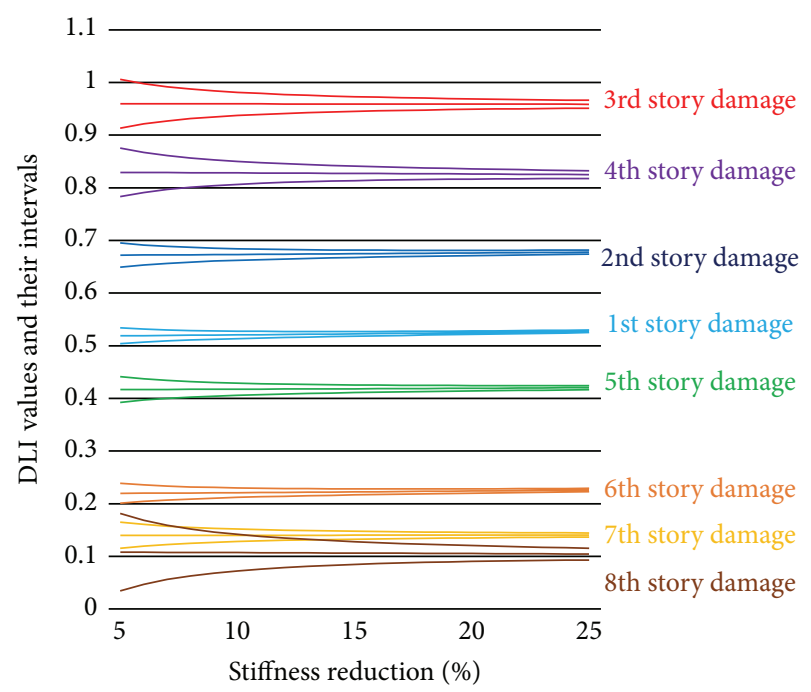

FIGURE 5: DLI values and their intervals within the reliability band $\pm 2 \sigma_{\mathrm{DLI}}$ of 8 -story structure.

obtained by (17) and are shown by the bold line in the center of each color.

The standard deviations of DLI were calculated by (22). The reliability band considering $\pm 2 \sigma_{\text {DLI }}$ was also calculated and is shown by the thin line above and below each color. The DLI values and their confidence intervals for 5\% to $25 \%$ of stiffness reduction at each story displayed by the different colors are presented in Figures 4 and 5 for the three- and eight-story shear structures, respectively.

To show the applicability of the proposed method to shear structures with variable stiffness, we used a five-story structure with different stiffnesses $\left(k_{1}=1.3 \times 10^{3} \mathrm{MN} / \mathrm{m}\right.$, $k_{2}=0.95 k_{1}, k_{3}=0.9 k_{1}, k_{4}=0.85 k_{1}$, and $\left.k_{5}=0.8 k_{1}\right)$.

The damping ratio for each mode was chosen to be $3 \%$. The data sampling frequency was $200 \mathrm{~Hz}$. The undamaged natural frequencies of the structure are 1.58 and $4.49 \mathrm{~Hz}$ for the 1st and 2nd modes, respectively. Damage was introduced by reducing the story stiffness of each story from 5\% to $25 \%$. The DLI values and their standard deviations were calculated and are presented in Figure 6 . The reliability band is $\pm 2 \sigma_{\mathrm{DLI}}$.

From Figures 3 to 6 , we can observe the following.

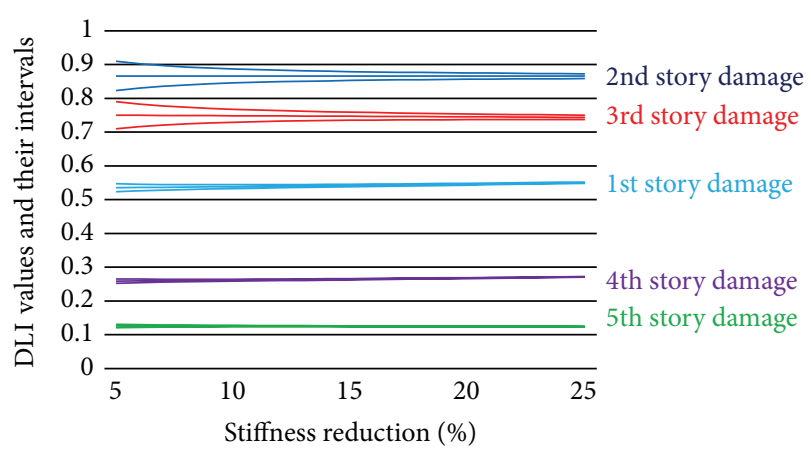

FIGURE 6: DLI values and their intervals within the reliability band $\pm 2 \sigma_{\text {DLI }}$ of 5-story structure with different stiffnesses.

(i) The DLI values (shown by the bold line in the center of each color) are stable at each damaged story and insensitive to the level of damage. The severity of structural damage cannot be detected.

(ii) The variations in DLI values (shown by the two thin lines above and below each color) depend on the stiffness reduction values. For smaller stiffness reductions, the variances of DLI values are larger.

(iii) The DLI value of an unknown data set of each structure should generally be within the reliability band of each color for each damaged location. In the eight-story structure case, the free-end stories (7th and 8th) damaged cases were misclassified when the damage level was less than $15 \%$, as shown in Figure 5 . Hence, a case study of tall buildings should be considered in the next step.

(iv) This proposed method is also applicable to shear structures with variable stiffness, as observed in Figure 6.

It is noted that the existence of the damage is determined by the change of the first and second natural frequencies. For example, we can set the trigger level of $2 \%$ for the above process. If the first or second natural frequency exceeds this level, we assume there exists damage in the structure.

\section{Experimental Verification}

A series of experiments on a five-story steel model (Figure 7(a)) was performed to verify the proposed method. The mass of each story was provided by aluminum slabs $(2.43 \mathrm{~kg})$. The columns were made from bronze plate springs $\left(0.0025 \times 0.03 \times 0.24 \mathrm{~m}^{3}\right)$. The structure was initially healthy with all the original columns intact (type 0 ). Damage was simulated in the experiment by replacing two original columns with two weaker ones. Three types of weaker columns were used in the test (Figure 7(b)).

(i) Type 1: $0.003 \times 0.014 \times 0.24 \mathrm{~m}^{3}(22 \%$ of stiffness reduction).

(ii) Type 2: $0.003 \times 0.010 \times 0.24 \mathrm{~m}^{3}(30 \%$ of stiffness reduction). 


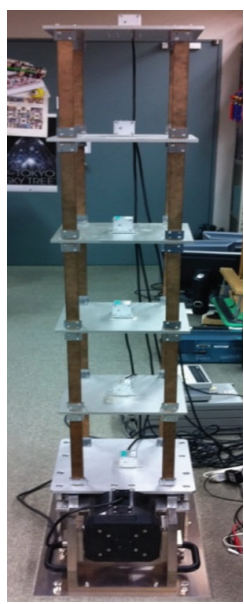

(a)

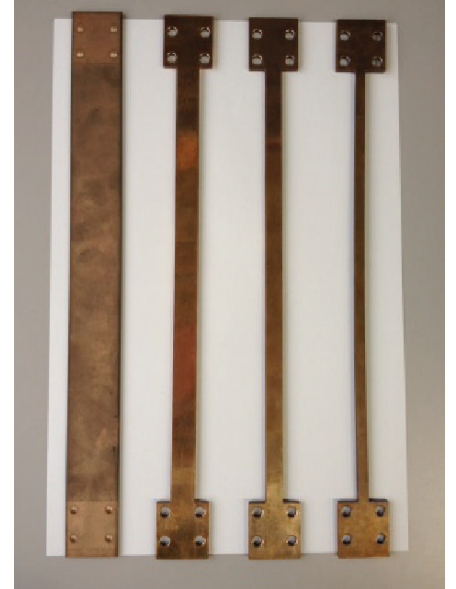

(b)
Figure 7: Experimental setup of five-story steel model (a) with undamaged (type 0 ) and damaged columns of types 1, 2, and 3 (b).

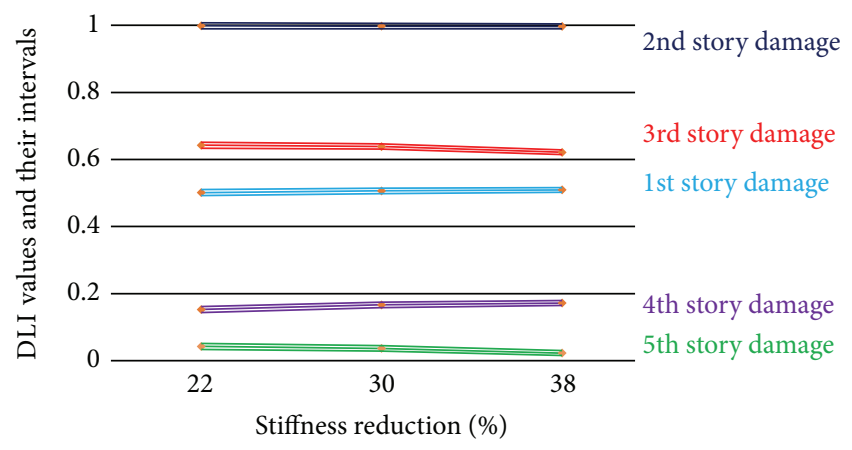

FIGURE 8: DLI values and their intervals within the reliability band $\pm 2 \sigma_{\text {DLI }}$ of experimental model.

(iii) Type 3: $0.003 \times 0.006 \times 0.24 \mathrm{~m}^{3}$ (38\% of stiffness reduction).

In total, data were collected for the undamaged state and fifteen $(3 \times 5=15)$ damaged cases.

The basement of the structure was set on bearings so that the structure could experience ground motion. The force input to the structure was provided with an electrodynamic shaker. One acceleration sensor was installed in the basement to measure the ground motion. Five other sensors, one installed on each floor plate, were used to measure the model's acceleration responses.

First, the structure was measured in the undamaged state with all the original columns. The shaker provided the force input to the structure in order to generate the acceleration data.

Next, two columns of each story were replaced by two weaker columns to simulate a single-story damage case. The shaker provided the same excitation again, and the accelerometers measured the acceleration data of the damaged structures. Although accelerometers were installed on all stories, only the data from the top and bottom sensors were used to obtain the modal frequencies.
The modal frequencies were calculated from the time histories of the vibration experiments by using the subspace identification method devised by Verhaegen and Dewilde in [19]. The undamaged natural frequencies of the structure were obtained at $2.23 \mathrm{~Hz}$ and $6.91 \mathrm{~Hz}$ for the 1st and $2 \mathrm{nd}$ modes, respectively.

Figure 8 shows the DLI values and their reliable bands in the experimental verifications, with the standard deviation of natural frequency $1 \%$. As the obtained frequencies with 2 digits of precision, the proposed method is acceptable for detecting the location of damage by these DLI patterns. From Figure 8, we can also see that the DLI values were stable when the damage level were $22 \%, 30 \%$, and $38 \%$, so that they can be used to detect the damage location. Therefore, we may conclude that the proposed method is applicable to realistic problems.

\section{Conclusion}

A method of determining the damage location in shear structures using the changes in only two first natural frequencies was proposed. Natural frequencies decrease because of damage, and these changes can be used to identify the location of damage with our proposed Damage Location Index (DLI). This DLI, based on two natural frequencies for undamaged and damaged states, is stable for each single damaged story. As we need only two first natural frequencies, two vibration sensors are enough to obtain modal frequencies: one on the ground detecting the input and the other on the roof detecting the output. If the input lasts for a long time and the spectrum is flat, we may identify those parameters using the output data without any input information. Thus, in such a case, only one sensor is enough.

The uncertainty associated with system identification methods for obtaining natural frequencies was also carefully considered and the confidence intervals of DLI values are acceptable. Some simulations and experiments on shear structures were conducted to verify the performance of the proposed method with single damage. In the near future, research on multiple damage to more than one story should be developed.

\section{Conflict of Interests}

The authors declare that there is no conflict of interests regarding the publication of this paper.

\section{Acknowledgments}

This work was supported in part by a Grant-in-Aid no. 22310103 (PI: Akira Mita) and Grant-in-Aid to the Global Center of Excellence Program for the "Center for Education and Research of Symbiotic, Safe and Secure System Design" from the Ministry of Education, Culture, Sport, Science and Technology of Japan. 


\section{References}

[1] A. Mita, Structural Dynamic for Health Monitoring, Sankeisha, Nagoya, Japan, 2003.

[2] J. Zhao and J. T. DeWolf, "Sensitivity study for vibrational parameters used in damage detection," Journal of Structural Engineering, vol. 125, no. 4, pp. 410-416, 1999.

[3] O. S. Salawu, "Detection of structural damage through changes in frequency: a review," Engineering Structures, vol. 19, no. 9, pp. 718-723, 1997.

[4] S. Doebling, Damage Identification and Health Monitoring of Structural and Mechanical Systems from Changes in Their Vibration Characteristics: A Literature Review, Los Alamos National Laboratory, Los Alamos, NM, USA, 1996.

[5] A. Mita and H. Hagiwara, "Quantitative damage diagnosis of Shear structures using Support Vector Machine," KSCE Journal of Civil Engineering, vol. 7, no. 6, pp. 683-689, 2003.

[6] N. Stubbs, T. H. Broome, and R. Osegueda, "Nondestructive construction error detection in large space structures," AIAA Journal, vol. 28, no. 1, pp. 146-152, 1990.

[7] Z. Xing and A. Mita, "A substructure approach to local damage detection of shear structure," Structural Control and Health Monitoring, vol. 19, no. 2, pp. 309-318, 2012.

[8] J. Sidhu and D. J. Ewins, "Correlation of finite and model test studies of a practical structure," in Proceeding of the 2nd International Modal Analysis Conference, pp. 756-762, Society for Experimental Mechanics, Orlando, Fla, USA, February 1984.

[9] H. P. Zhu and Y. L. Xu, "Damage detection of mono-coupled periodic structures based on sensitivity analysis of modal parameters," Journal of Sound and Vibration, vol. 285, no. 1-2, pp. 365-390, 2005.

[10] A. Messina, E. J. Williams, and T. Contursi, "Structural damage detection by a sensitivity and statistical-based method," Journal of Sound and Vibration, vol. 216, no. 5, pp. 791-808, 1998.

[11] E. P. Carden and A. Mita, "Challenges in developing confidence intervals on modal parameters estimated for large civil infrastructure with stochastic subspace identification," Structural Control and Health Monitoring, vol. 18, no. 1, pp. 53-78, 2011.

[12] W. Gao, "Interval natural frequency and mode shape analysis for truss structures with interval parameters," Finite Elements in Analysis and Design, vol. 42, no. 6, pp. 471-477, 2006.

[13] W. Gao, "Natural frequency and mode shape analysis of structures with uncertainty", Mechanical Systems and Signal Processing, vol. 21, no. 1, pp. 24-39, 2007.

[14] P. Moser and B. Moaveni, "Environmental effects on the identified natural frequencies of the Dowling Hall Footbridge," Mechanical Systems and Signal Processing, vol. 25, no. 7, pp. 2336-2357, 2011.

[15] K. He and W. D. Zhu, "Structural damage detection using changes in natural frequencies: theory and applications," Journal of Physics: Conference Series, vol. 305, no. 1, Article ID 012054, 2011.

[16] D. Muria-Vila, L. Fuentes, and R. Gonzalez, "Uncertainties in the estimation of natural frequencies of vibration in buildings of Mexico City," Informacion Tecnologica, vol. 11, no. 3, pp. 177$184,2000$.

[17] J. F. Clinton, S. C. Bradford, T. H. Heaton, and J. Favela, "The observed wander of the natural frequencies in a structure," Bulletin of the Seismological Society of America, vol. 96, no. 1, pp. 237-257, 2006.
[18] V. Lindberg, "Uncertainties and error propagation," 2008, http://www.rit.edu/cos/uphysics/uncertainties/Uncertaintiespart2.html.

[19] M. Verhaegen and P. Dewilde, "Subspace model identification part 1: the output-error state-space model identification class of algorithms," International Journal of Control, vol. 56, no. 5, pp. 1187-1210, 1992. 

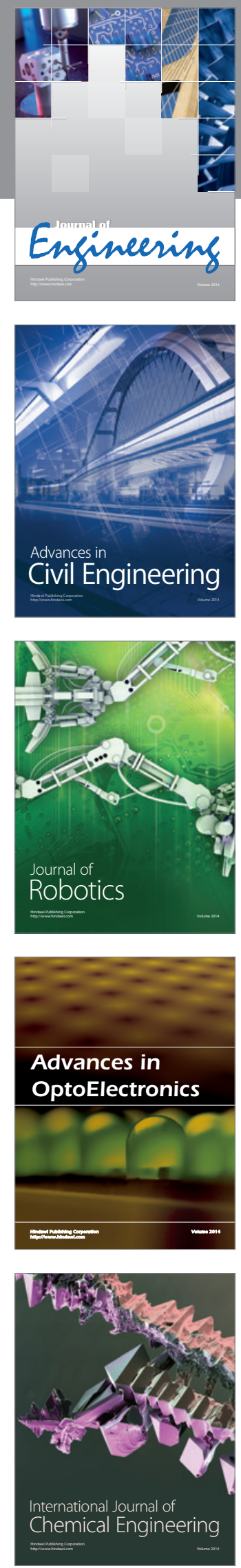

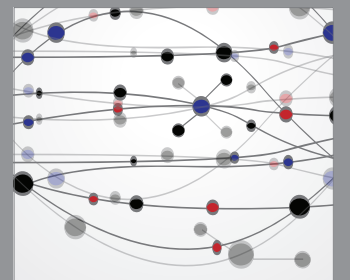

The Scientific World Journal
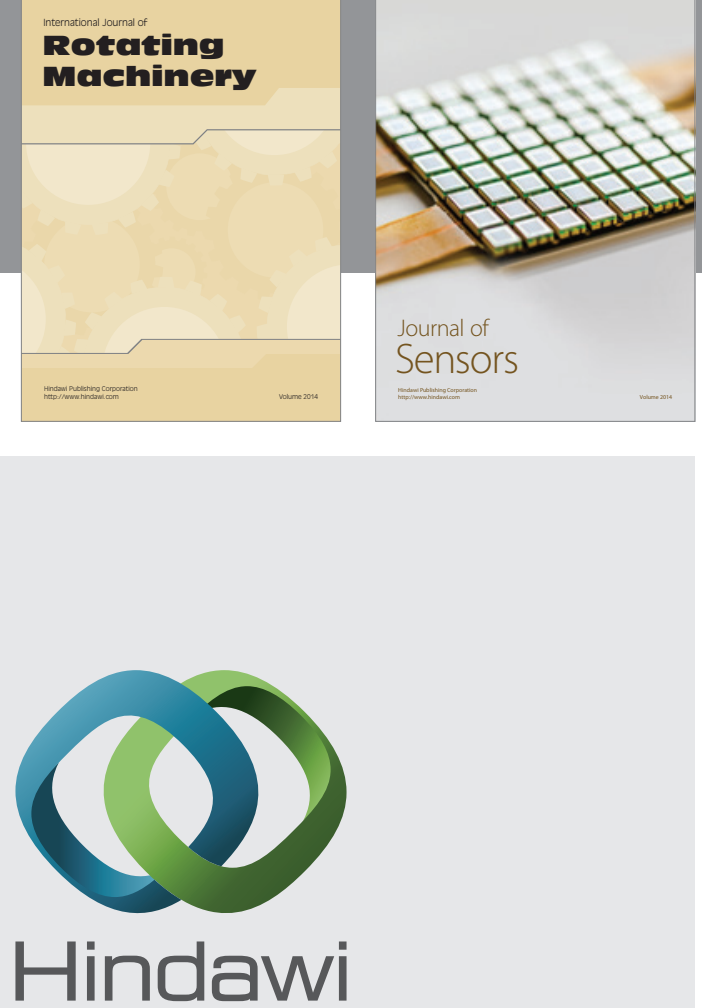

Submit your manuscripts at http://www.hindawi.com
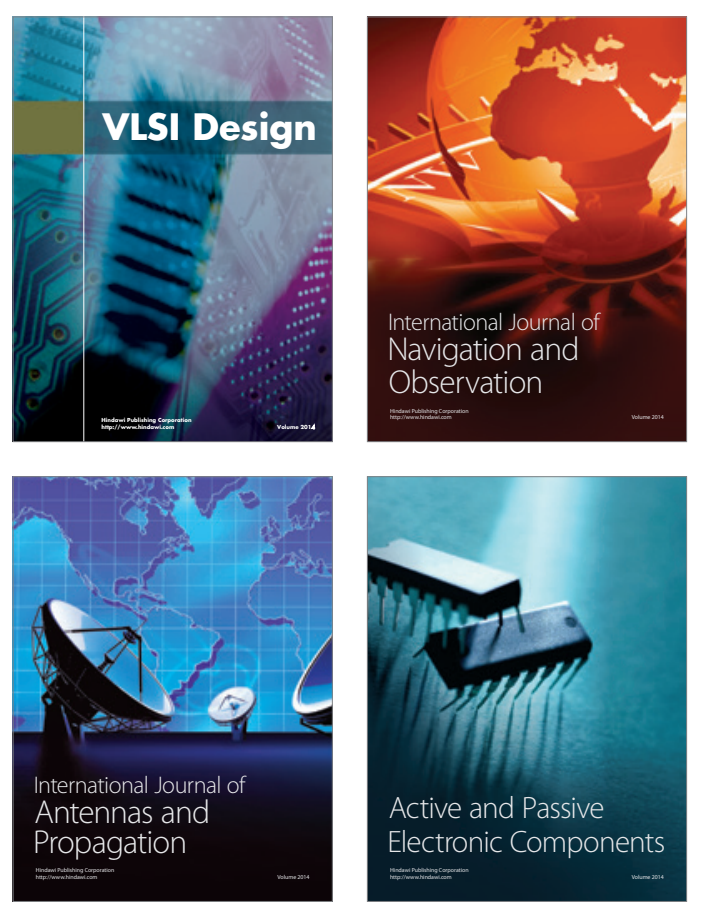
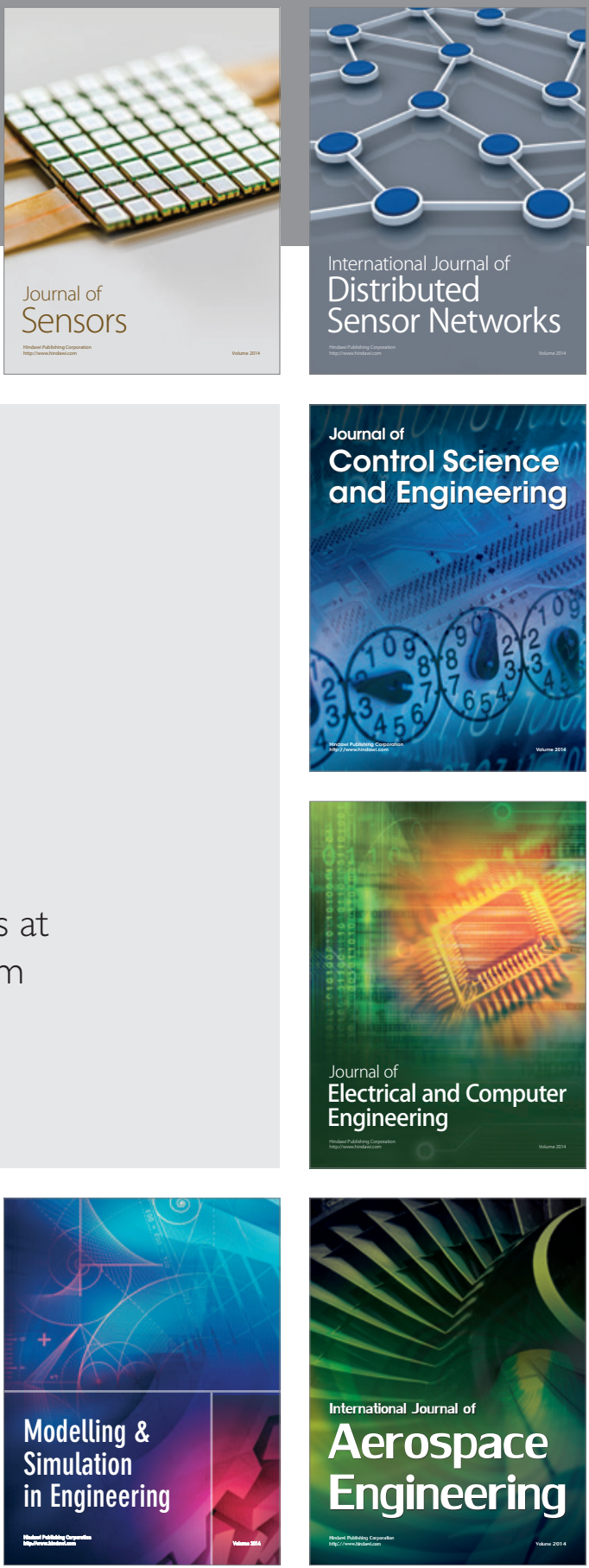

Journal of

Control Science

and Engineering
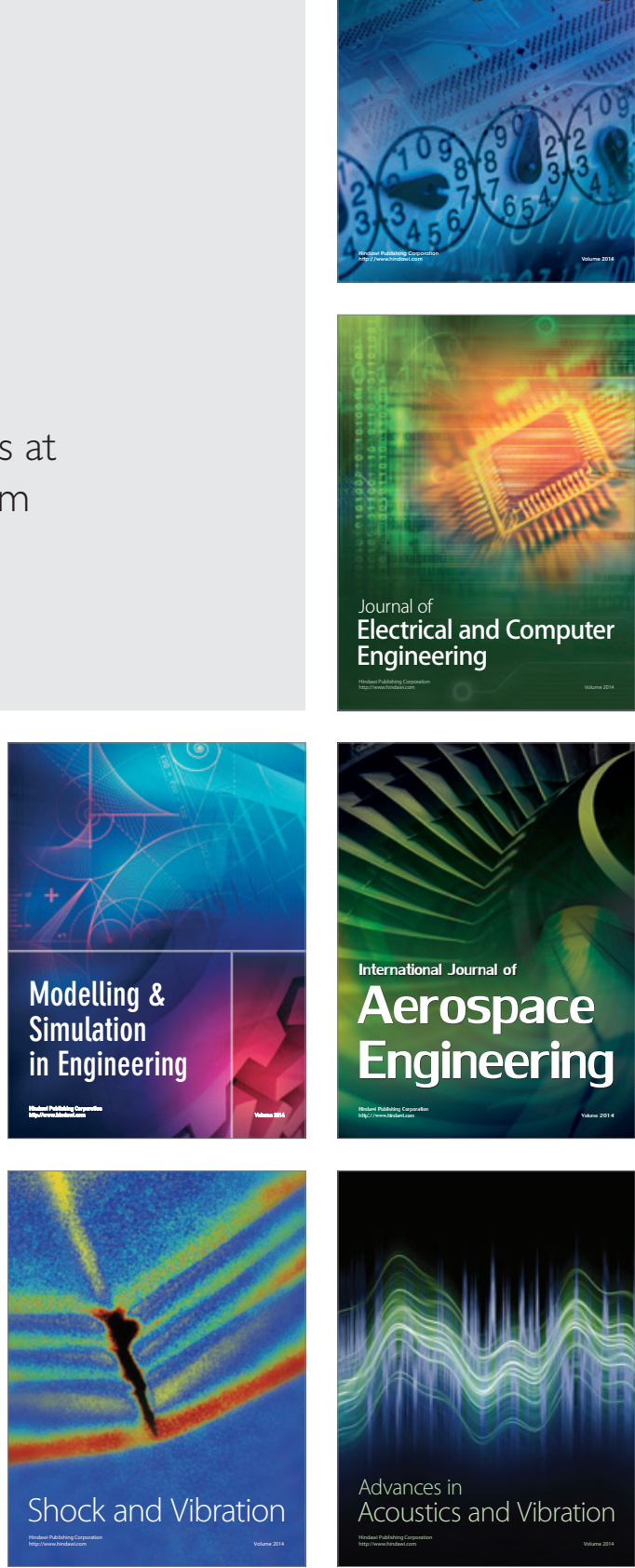